\title{
Washed microbiota transplantation vs. manual fecal microbiota transplantation: clinical findings, animal studies and in vitro screening
}

\author{
Ting Zhang ${ }^{1,2}$, Gaochen $\mathrm{Lu}^{1,2}$, Zhe Zhao ${ }^{3}$, Yafei Liu ${ }^{1,2}$, Quan Shen ${ }^{4}$, Pan $\mathrm{Li}^{1,2}$, Yaoyao Chen ${ }^{1,2}$, Haoran Yin ${ }^{3}$, \\ Huiquan Wang $^{3}$, Cicilia Marcella ${ }^{1,2}$, Bota Cui ${ }^{1,2}$, Lei Cheng ${ }^{5,6}$, Guozhong $\mathrm{Ji}^{1,2}$, Faming Zhang ${ }^{1,2,7 凶}$ \\ ${ }^{1}$ Medical Center for Digestive Diseases, The Second Affiliated Hospital of Nanjing Medical University, Nanjing 210011, China \\ ${ }^{2}$ Key Lab of Holistic Integrative Enterology, Nanjing Medical University, Nanjing 210011, China \\ 3 Tianjin Key Laboratory of Optoelectronic Detection Technology and Systems, Tianjin 300387, China \\ ${ }^{4}$ Department of Microbiology, School of Medicine, Jiangsu University, Zhenjiang 212013, China \\ ${ }^{5}$ Biogas Institute of Ministry of Agriculture and Rural Affairs, Chengdu 610041, China \\ ${ }^{6}$ Center for Anaerobic Microbial Resources of Sichuan Province, Chengdu 610041, China \\ ${ }^{7}$ Division of Microbiotherapy, Sir Run Run Shaw Hospital, Nanjing Medical University, Nanjing 211166, China \\ $\triangle$ Correspondence: fzhang@njmu.edu.cn (F. Zhang)
}

Received November 8, 2019 Accepted December 3, 2019

\begin{abstract}
Fecal microbiota transplantation (FMT) by manual preparation has been applied to treat diseases for thousands of years. However, this method still endures safety risks and challenges the psychological endurance and acceptance of doctors, patients and donors. Population evidence showed the washed microbiota preparation with microfiltration based on an automatic purification system followed by repeated centrifugation plus suspension for three times significantly reduced FMT-related adverse events. This washing preparation makes delivering a precise dose of the enriched microbiota feasible, instead of using the weight of stool. Intraperitoneal injection in mice with the fecal microbiota supernatant obtained after repeated centrifugation plus suspension for three times induced less toxic reaction than that by the first centrifugation following the microfiltration. The toxic reactions that include death, the change in the level of peripheral white blood cells, and the proliferation of germinal center in secondary lymphoid follicles in spleen were noted. The
\end{abstract}

Ting Zhang, Gaochen Lu and Zhe Zhao have contributed equally to this study.

Electronic supplementary material The online version of this article (https://doi.org/10.1007/s13238-019-00684-8) contains supplementary material, which is available to authorized users. metagenomic next-generation sequencing (NGS) indicated the increasing types and amount of viruses could be washed out during the washing process. Metabolomics analysis indicated metabolites with pro-inflammatory effects in the fecal microbiota supernatant such as leukotriene B4, corticosterone, and prostaglandin G2 could be removed by repeated washing. Near-infrared absorption spectroscopy could be served as a rapid detection method to control the quality of the washingprocess. In conclusion, this study for the first time provides evidence linking clinical findings and animal experiments to support that washed microbiota transplantation (WMT) is safer, more precise and more quality-controllable than the crude FMT by manual.

KEYWORDS fecal microbiota transplantation, washed microbiota transplantation, adverse event, safety, infection, virus, metabolomics, spectroscopy, transplant

\section{INTRODUCTION}

Fecal microbiota transplantation (FMT), an effective method of reconstructing the overall gut microbiota of patients, has a wide range of therapeutic indications (Zhang et al., 2018; Allegretti et al., 2019). It has been recommended for the treatment of recurrent Clostridioides difficile infection (CDI) in the clinical guideline, consensus and joint-experts 
recommendation (McDonald et al., 2018; $\mathrm{Ng}$ et al., 2019). Increasing published randomized controlled trials (RCTs) and real-world studies demonstrated that FMT is of important therapeutic value in diseases beyond CDI, such as ulcerative colitis (UC) (Moayyedi et al., 2015; Paramsothy et al., 2017; Costello et al., 2019; Ding et al., 2019; Sood et al., 2019), Crohn's disease (CD) (Wang et al., 2018), hepatic encephalopathy (Bajaj et al., 2017), and autism (Kang et al., 2017). Tracing the origins of FMT in the world medical history, the earliest record of FMT for the treatment of human diseases began at least in the 4th century in China (Zhang et al., 2012). However, up to now, the protocol introduced in published consensuses is merely mixing the stool with saline in a blender by the manual method (Cammarota et al., 2017; Konig et al., 2017; $\mathrm{Ng}$ et al., 2019). The fecal matter actually includes many particles that even caused obstruction through colonic transendoscopic enteral tube (Wang et al., 2019). The dose for delivering FMT is mainly based on the donors' fecal weight, instead of the precise volume or amount of microbiota (Cammarota et al., 2019).

The recent surveys on doctors, medical students, donors, and patients show that they have a negative perception towards FMT, especially its crude methods (Zipursky et al., 2014; Ma et al., 2017; Park et al., 2017; McSweeney et al., 2019; Wu et al., 2019). Doctors are generally less willing to recommend FMT than patients, and one reason is that they need to have contact with feces and prepare the feces by manual method (Brandt, 2012; Zipursky et al., 2012, 2014; Ma et al., 2017). Based on a survey of 241 patients, 22\% think it is "dirty/unsanitary", and this is a possible reason why they might not be willing to receive FMT (Park et al., 2017). Negative perceptions of society significantly limit the use of FMT. Importantly, DeFilipp et al. (2019) recently reported that two patients developed extended-spectrum beta-lactamase (ESBL)-producing bacteremia after FMT. This highlighted the importance to improve the safety of FMT. Therefore, the FMT-standardization Study Group stated that it is time to leave the manual FMT behind and move FMT standardization forward (Zhang et al., 2018).

An automatic preparation method for enriching microbiota from feces has been developed and is being used in serial FMT centers in China since 2014 (Cui et al., 2015; Cui et al., 2016; Qi et al., 2018; Wang et al., 2018; Ding et al., 2019; Huang et al., 2019). This method, originally designed on the concept of washed microbiota preparation is based on the automatic microfiltration machine (GenFMTer, Nanjing, China) and the following repeated centrifugation plus suspension with support from specific facilities. Based on the patients who underwent either washed microbiota transplantation (WMT) or crude FMT in the same FMT center with the same clinical workflow, the multiple factors analysis demonstrated that the washed microbiota preparation is an independent factor contributing to the decreased adverse events (AEs) from $38.7 \%$ to $14.4 \%$ in patients with UC(Ding et al., 2019). The rate of AEs decreased significantly from $21.7 \%$ in crude $\mathrm{FMT}$ to $8.7 \%$ in WMT in patients with CD
(Wang et al., 2018). Importantly, the washed microbiota preparation did not affect the efficacy in both populations with UC and CD, compared with that by manual preparation for fecal microbiota (Wang et al., 2018; Ding et al., 2019).

However, there is lacking reported theoretical evidence for understanding the reason why washed microbiota preparation is safer and better than the commonly used crude method for fecal microbiota preparation. This study aimed to investigate how the washing preparation is better than the manual preparation for FMT.

\section{RESULTS}

Washed microbiota preparation decreased the FMTrelated AEs

Clinical data from a total of 970 patients (478 with UC and 492 with CD) who underwent FMT was recorded into China microbiota transplantation system (CMTS) for over a oneyear follow-up (Fig. 1). Figure 2 showed the rate of AEs decreased significantly in patients with UC who underwent an automatic method for the preparation of fecal microbiota than those who experienced a manual method $(38.7 \%$ vs. $12.3 \%, P<0.001)$. In CD, the rate of AEs in patients who underwent a manual method was $21.7 \%$, which was significantly higher than the rate of $4.26 \%$ in those who underwent an automatic method $(P<0.001)$. The fever after FMT significantly decreased from $19.35 \%$ in manual preparation to $5.15 \%$ in automatic preparation for fecal microbiota $(P=$ $0.001)$.

The relationship between fecal weight and the amount of enriched microbiota in donors

Following the automatic preparation for washing microbiota, we also explored the relationship between fecal weight and the amount of enriched microbiota. As shown in Fig. 2D, the fecal weight was correlated with the amount of enriched washed microbiota (95\% Cl, 0.61-0.68, $P<0.0001$ ), but $r$ was 0.65 . Even for the different defecation samples from the same donor, the relationship between the fecal weight and amount of microbiota was not well satisfactory (Fig. 2E). The correlation coefficient $r$ in adult donors is $0.65(95 \% \mathrm{Cl}, 0.57-$ $0.72, P<0.0001)$, while it is 0.36 in children donors $(95 \% \mathrm{Cl}$, $0.10-0.57, P=0.0085)$. The dose of enriched microbiota for adult patients and children over than 7 years is $50 \mathrm{~cm}^{3}$ of microbiota precipitation for the regular treatment and the dose of enriched microbiota for children from 1 to 7 year-old ranged $10-50 \mathrm{~cm}^{3}$. The volume ratio of final precipitation/ vector solution is 1:2 for making suspension as fresh use or frozen use. For the convenience in communications during medical practice, we defined one unit to replace $10 \mathrm{~cm}^{3}$ microbiota precipitation $\left(\sim 1.0 \times 10^{13}\right.$ bacteria $)$ in the medical record in CMTS and clinical flow. This measurement was confirmed in our previous experiments and it is the first time to open to public. 


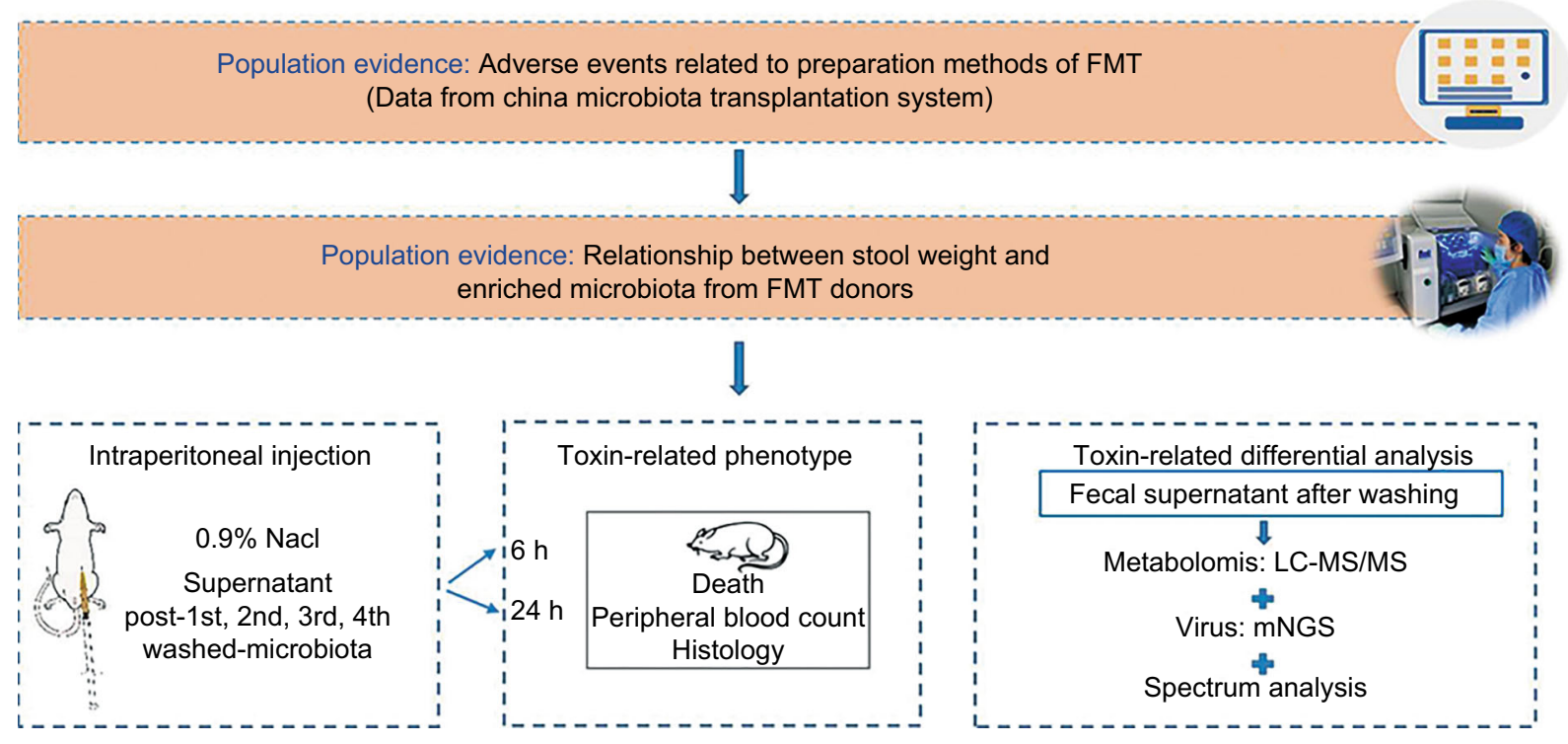

Figure 1. Flow chart of the study.

Toxic responses in mice caused by intraperitoneal injection of fecal microbiota supernatant

To evaluate the role of clinical laboratory washing process on reducing the rate of $\mathrm{AEs}$ after WMT, the different supernatants obtained after one to four times of washing were used for intraperitoneal injection in mice. As shown in Fig. $2 \mathrm{H}$, the death rate in the group of Supernatant 1 was $70 \%$, and no death was observed in the groups of Supernatant 2, Supernatant 3, and Supernatant 4 at $24 \mathrm{~h}$ after intraperitoneal injection. Decreased activity, lower temperature, and chills were observed in the group of Supernatant 1 , but no similar symptoms were observed in other groups.

\section{Fecal microbiota supernatant induced peripheral blood cells changes at $6 \mathrm{~h}$ after intraperitoneal injection}

After $6 \mathrm{~h}$ of intraperitoneal injection, the number of white blood cells (WBC) in the group of Supernatant 1 and 2 was significantly decreased compared with the group of normal saline $(P<0.001, P<0.001$, respectively) (Fig. 3). However, the number of WBC in the Supernatant 3 was close to that in the normal saline group $(7.70 \pm 1.87$ vs. $7.90 \pm 2.11, P>$ 0.05). No significant difference was observed in the WBC count between the Supernatant 3 and Supernatant 4 group.

Among all four groups, the changes in red blood cells (RBC) are consistent with the trend of WBC (Fig. 3). There was no difference in the number of RBC between the Supernatant 3 and the normal saline group. Similarly, no significant difference was observed in both RBC and platelets (PLT) between Supernatant 3 and Supernatant 4. Figure $\mathrm{S} 1$ showed that neutrophil (NEUT), percentage of NEUT, lymphocyte (LYM), percentage of LYM, NEUT to LYM ratio
(NLR), and PLT to LYM ratio (PLR) of the Supernatant 3 were all close to those in the normal saline group.

Fecal microbiota supernatant induced peripheral blood cells changes at $24 \mathrm{~h}$ after intraperitoneal injection

After $24 \mathrm{~h}$ of intraperitoneal injection, the number of WBC in the group of Supernatant 1 was significantly lower, while the number of RBC was higher than that in the group of the normal saline $(P<0.001, P<0.01$, respectively). But no significant difference was observed between the Supernatant 3 and the normal saline group for WBC, RBC, PLT, NEUT, percentage of NEUT, NLR, LYM and PLR (Fig. 4 and S2). At the same time, there was no difference in the number of WBC, RBC and PLT between the Supernatant 3 and Supernatant 4 group.

Comparison between different time points showed that the number of WBC at $24 \mathrm{~h}$ was significantly lower than that at $6 \mathrm{~h}$ after intraperitoneal injection of the Supernatant 1 $(1.74 \pm 0.1$ vs. $4.43 \pm 1.87, P=0.04)$ (Fig. 4). However, no significant decrease in WBC at $24 \mathrm{~h}$ was observed for Supernatant 3. Compared with the value at $6 \mathrm{~h}$, NEUT\% significantly decreased at $24 \mathrm{~h}$ in the Supernatant 3 group $(P=0.009)$. However, NEUT\% in the Supernatant 1 group did not differ significantly between $6 \mathrm{~h}$ and $24 \mathrm{~h}$.

\section{Fecal microbiota supernatant-caused pathological change of spleen in mice}

As shown in Fig. 5, in the group of Supernatant 1, we observed apparent neutrophils infiltration, which was marked as "++-+++". And multiple germinal centers of secondary lymphoid follicles were seen in the group of Supernatant 1 and the proliferation of secondary lymphoid follicles was 



$\mathrm{H}$

\begin{tabular}{|c|c|c|c|c|c|c|c|}
\hline \multirow{2}{*}{ Group } & \multicolumn{6}{|c|}{ Death in mice after i.p. of fecal supernatant } & \multirow{2}{*}{ Death rate $(\%)$} \\
\hline & $2 \mathrm{~h}$ & $4 \mathrm{~h}$ & $6 \mathrm{~h}$ & $8 \mathrm{~h}$ & $16 \mathrm{~h}$ & $24 \mathrm{~h}$ & \\
\hline $0.9 \% \mathrm{NaCl}$ & 0 & 0 & 0 & 0 & 0 & 0 & 0 \\
\hline Supernatant 1 & 0 & 0 & 0 & 0 & 3 & 4 & 70 \\
\hline Supernatant 2 & 0 & 0 & 0 & 0 & 0 & 0 & 0 \\
\hline Supernatant 3 & 0 & 0 & 0 & 0 & 0 & 0 & 0 \\
\hline Supernatant 4 & 0 & 0 & 0 & 0 & 0 & 0 & 0 \\
\hline
\end{tabular}

Figure 2. Evidence related to fecal microbiota preparation from human population to animal. (A-C) AEs related to manual and automatic preparation for fecal microbiota in patients. (D-F) Relationship between fecal weight and enriched microbiota from FMT donors. $(\mathrm{H})$ Death time and rate after intraperitoneal injection of fecal supernatant in mice (i.p., intraperitoneal injection). Statistical comparisons are performed using chi-square test; ${ }^{\star} P<0.05$, ${ }^{\star \star} P<0.01,{ }^{\star \star \star} P<0.001$. Correlation analysis was performed using Spearman correlation analysis. Data are presented as mean \pm standard deviation (SD).

marked as "+". In the group of Supernatant 3, the degree of neutrophils infiltration was "+-++", and there was no proliferation of primary follicles (-). No cavity or necrosis of spleen tissue and bacterial mass was observed in all groups.

\section{Differential screening of virus changes during the washing process}

In order to identify what viruses were washed out during washing process, the metagenomic next-generation sequencing (NGS) was used to differentiate the Supernatant 1 and the Supernatant 3. Compared with the Supernatant 1, the types and number of viruses tested by metagenomic
NGS of the Supernatant 3 showed an incremental trend (Fig. 6). Only one type of virus named pepper mild mottle virus was found in all five donors, and Tobacco mild green mosaic virus was found in four donors. The NGS reads of the Supernatant 3 also increased in these two viruses (Fig. 6C and $6 \mathrm{D}$ ). Figure $6 \mathrm{E}$ showed the fold change between the Supernatant 1 and the Supernatant 3 in the number of the top ten viruses. The fold change of the virus named watermelon mosaic virus ranked the top 1 , and its sequencing number in the Supernatant 3 was 40.17 times higher than that in the Supernatant 1.

Figure 7 showed the types and numbers of the top five viruses in each donor. Donor $C$ had the minimum virus 

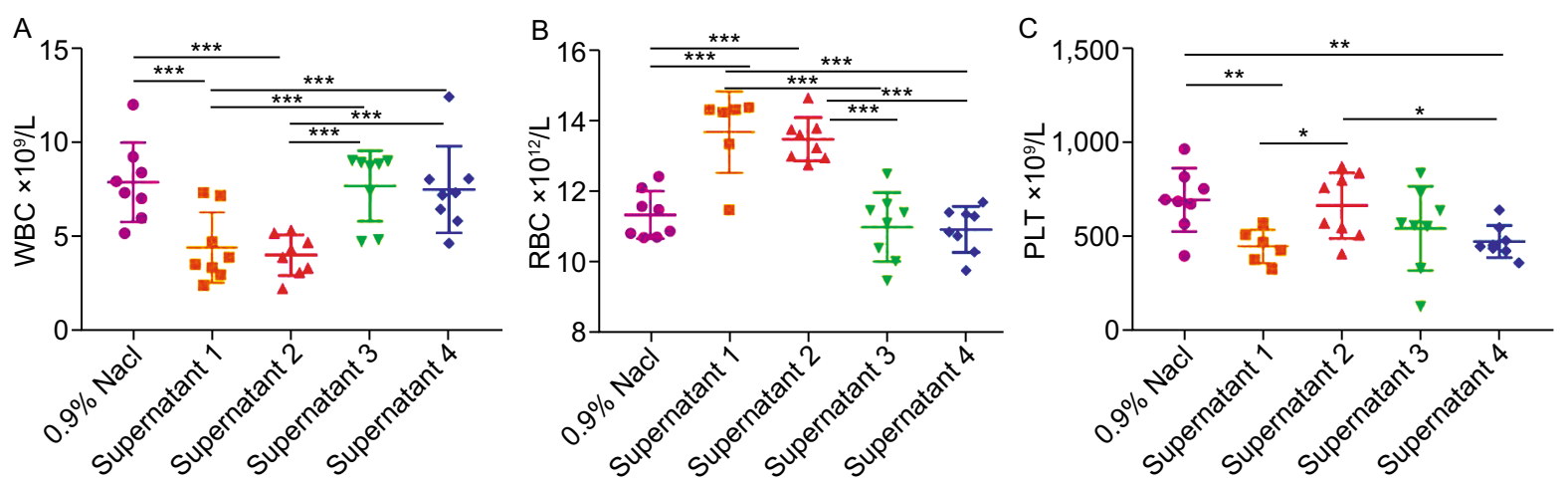

Figure 3. Changes of peripheral blood cells in the five groups of mice after $6 \mathrm{~h}$ of intraperitoneal injection of fecal microbiota supernatant. (A) Changes of WBC ( $n=8$ animals/group). (B) Changes of RBC ( $n=8$ animals/group). (C) Changes of PLT ( $n=8$ animals/group). Statistical comparisons are performed using one-way ANOVA; ${ }^{\star} P<0.05,{ }^{\star \star} P<0.01,{ }^{\star \star *} P<0.001$. Data are presented as mean $\pm \mathrm{SD}$.
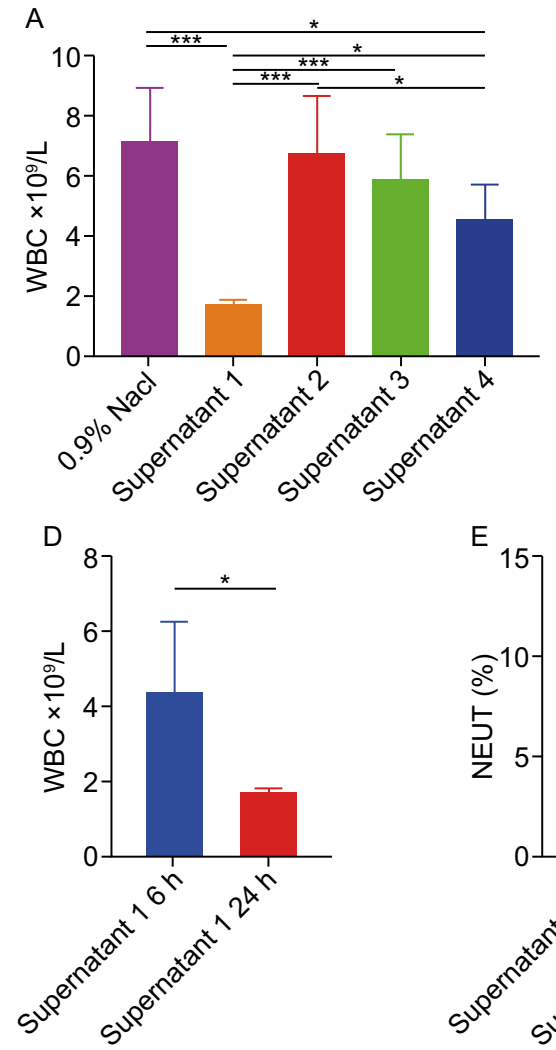

B
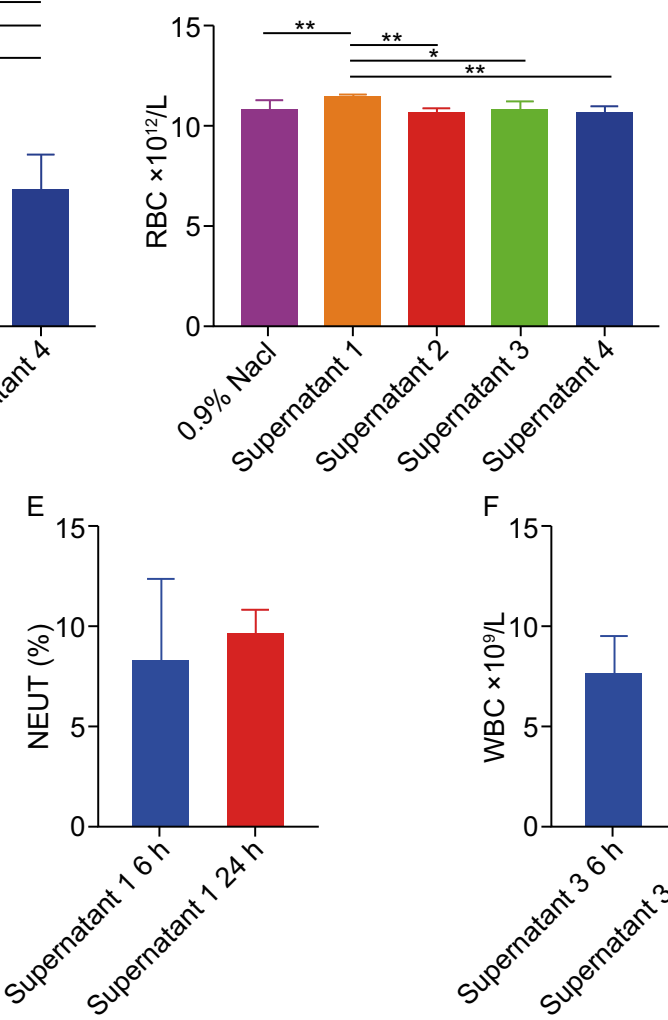

C
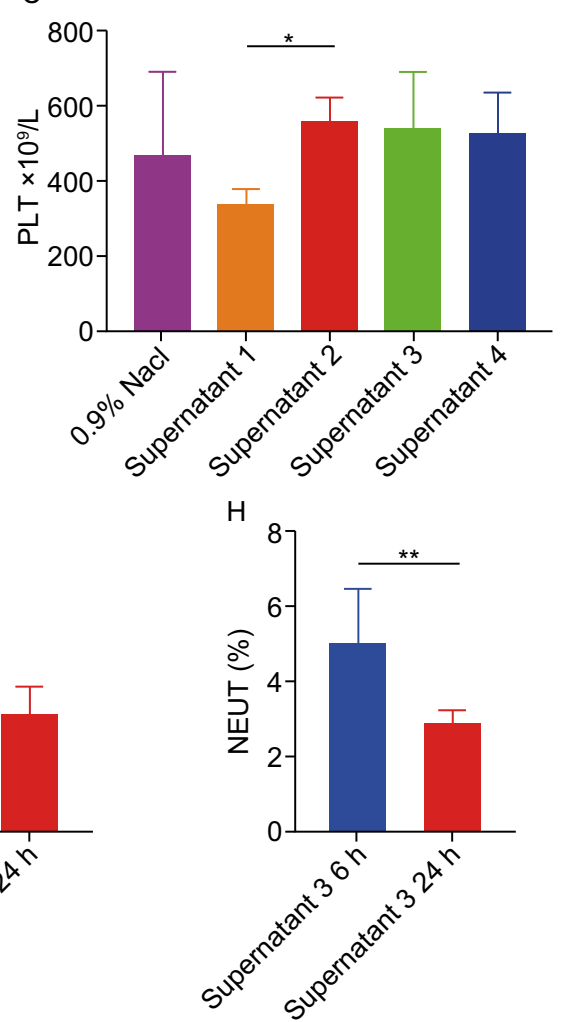

Figure 4. Changes of peripheral blood cells of mice after intraperitoneal injection of fecal microbiota supernatant. (A) Changes of WBC in the five groups of mice after $24 \mathrm{~h}$ (Supernatant $1, n=3$; other groups, $n=8$ ). (B) Changes of RBC in the five groups of mice after $24 \mathrm{~h}$ (Supernatant $1, n=3$; other groups, $n=8$ ). (C) Changes of PLT in the five groups of mice after $24 \mathrm{~h}$ (Supernatant $1, n=3$; other groups, $n=8$ ). (D) Changes of WBC at $6 \mathrm{~h}$ and $24 \mathrm{~h}$ after injection with Supernatant 1 (Supernatant $16 \mathrm{~h}$, $n=8$; Supernatant $124 \mathrm{~h}, n=3$ ). (E) Changes in the percentage of NEUT at $6 \mathrm{~h}$ and $24 \mathrm{~h}$ after injection with Supernatant 1 (Supernatant $16 \mathrm{~h}, n=8$; Supernatant $124 \mathrm{~h}, n=3)$. (F) Changes of WBC at $6 \mathrm{~h}$ and $24 \mathrm{~h}$ after injection with Supernatant 3 ( $n=$ 8/group). (H) Changes in the percentage of NEUT at $6 \mathrm{~h}$ and $24 \mathrm{~h}$ after injection with Supernatant 3 ( $n=8 /$ group). Statistical comparisons $(A-C)$ are performed using one-way ANOVA; statistical comparisons $(D-H)$ are performed using unpaired $t$-tests; ${ }^{*}<<$ $0.05,{ }^{* \star} P<0.01,{ }^{* \star *} P<0.001$. Data are presented as mean $\pm \mathrm{SD}$. 

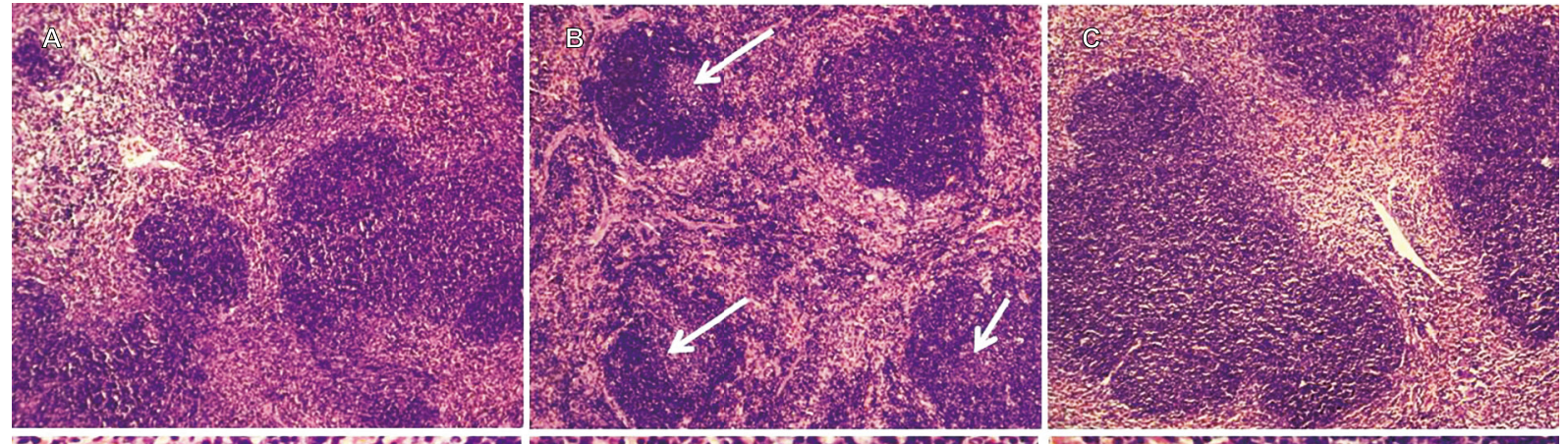

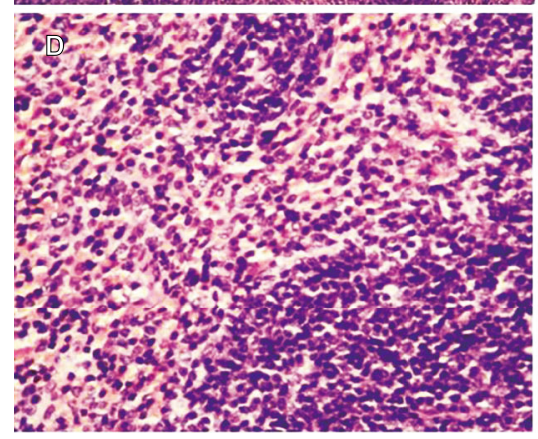

$0.9 \% \mathrm{NaCl}$

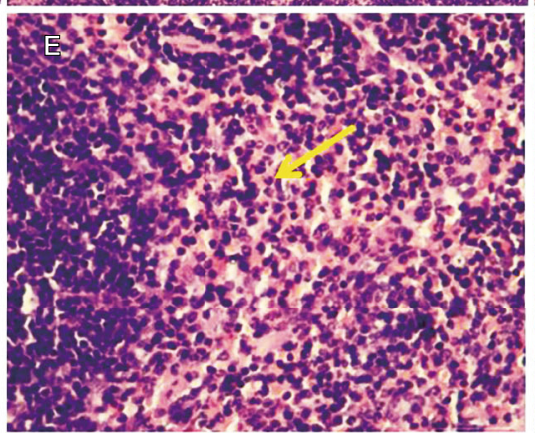

Supernatant 1

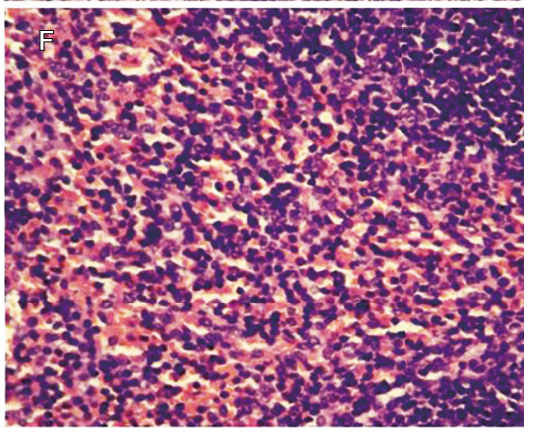

Supernatant 3

Figure 5. HE staining of spleen in mice at $6 \mathrm{~h}$ after intraperitoneal injection with the fecal microbiota supernatant. (A) No proliferation of primary follicles was observed in the group of normal saline $(\times 200)$. (B) The proliferation of secondary follicles was observed in the group of Supernatant $1(\times 200)$. (C) No proliferation of primary follicles was observed in the group of Supernatant 3 $(\times 200)$. (D) NEUT infiltration in the group of normal saline $(\times 400)$. (E) NEUT infiltration in the group of Supernatant $1(\times 400)$. (F) NEUT infiltration in the group of Supernatant $3(\times 400)$. White arrow noted: germinal center of secondary follicles; yellow arrow noted: NEUT infiltration.

sequencing reads both in the Supernatant 1 and the Supernatant 3 . Donor $E$ had the maximum virus sequencing reads in the Supernatant 1 , and Donor $A$ had the maximum one in the Supernatant 3.

\section{Differential screening of metabolites during the washing process}

In order to identify what metabolites were washed out during washing process, the method of LC-MS was used to differentiate the Supernatant 1 and the Supernatant 3 . The principle component analysis (PCA) and partial least squares method-discriminant analysis (PLS-DA) showed that there was a significant difference in overall metabolite composition between Supernatant 1 and Supernatant 3. A total of 78 differential metabolites were identified. Compared with the Supernatant 3, 31 differential metabolites were significantly up-regulated and 47 differential metabolites were significantly down-regulated in the Supernatant 1 ( $q<0.05)$. The up-regulated metabolites in the Supernatant 1 included leukotriene B4 (LtB4), corticosterone (CORT), prostaglandin G2 (PGG2), 5-hydroxyindole-3-acetic acid (5-HIAA) and so on. And the metabolites of tretinoin, stearidonic acid, caffeic acid, tyrosol, aspirin, 4-hydroxy-2-nonenal, resveratrol, chloral hydrate, paracetamol, calcitriol, adenosine, etc. were up-regulated in the Supernatant 3. The results of cluster analysis of differential metabolites were shown in Fig. 8B. Based on the Kyoto encyclopedia of genes and genomes (KEGG) database, the significantly enriched metabolic pathways on differential metabolites were shown in Fig. 8F, such as inflammatory mediator regulation of transient receptor potential (TRP) channels, PPAR signaling pathway, phenylalanine metabolism $(P<0.05)$.

\section{Differential testing by near-infrared absorption (NIRS) spectroscopy}

In order to develop a convenient technique to quickly verify the quality of washing microbiota, NIRS spectroscopy was applied to analyze the changes of light intensity and absorbance after different times of washing. In this NIRS experiment, the normal saline was used as a standard control and its transmission light intensity was the highest, as well as the absorbance was the smallest as compared with other samples (Fig. 9). As the washing times increasing, the light intensity of the supernatant increased consistently $(P<$ 0.001 ), which was getting closer to that of the normal saline. There was a significant difference in the light intensity between the Supernatant 1 and the Supernatant $3(P<$ 0.001). When it comes to the absorbance of light, 

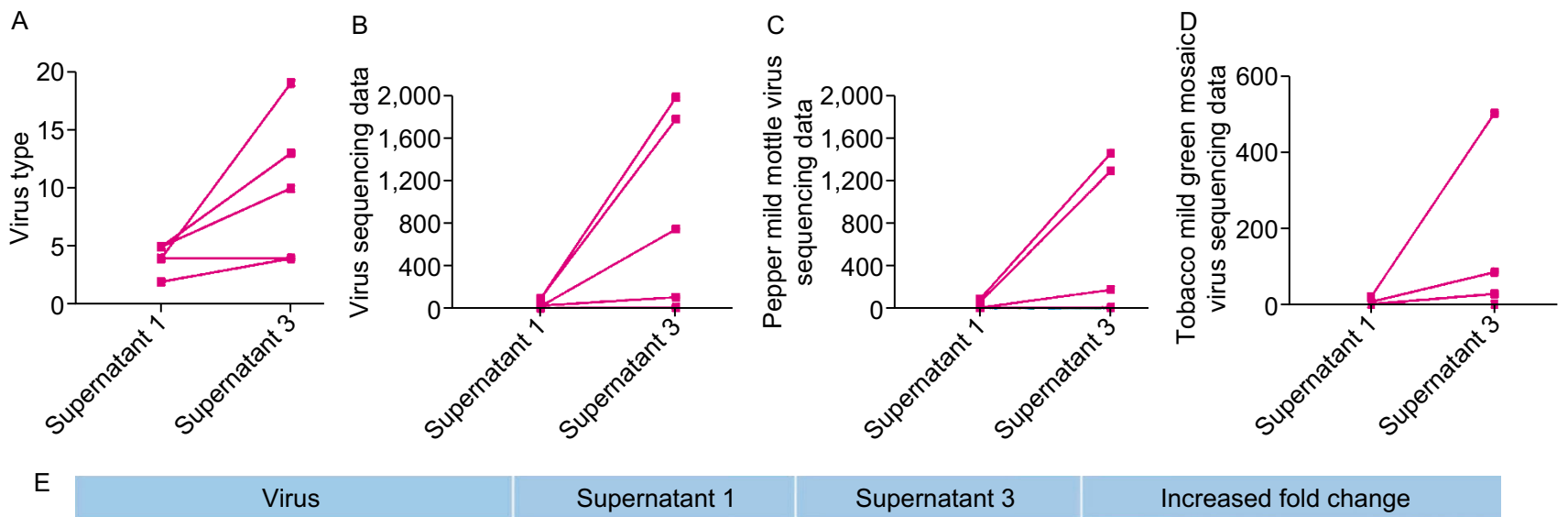

\begin{tabular}{|c|}
\hline Virus \\
\hline Watermelon mosaic virus \\
\hline Cherry green ring mottle virus \\
\hline Paprika mild mottle virus \\
\hline Oryza sativa endorna virus \\
\hline Tobacco mild green mosaic virus \\
\hline Garlic latent virus \\
\hline Melon aphid borne yellows virus \\
\hline Spinach latent virus \\
\hline Brassica yellows virus
\end{tabular}

Pepper mild mottle virus

\section{Supernatant 1}

Supernatant 3

\begin{tabular}{c|c|}
241 \\
116 \\
\hline 28 \\
\hline
\end{tabular}

\begin{tabular}{|c|}
\hline Increased fold change \\
\hline 40.17 \\
\hline 28.67 \\
\hline 28.00 \\
22.40 \\
22.00 \\
17.60 \\
\hline 9.11 \\
\hline 6.85 \\
\hline 3.33 \\
\hline 1.23 \\
\hline
\end{tabular}

Figure 6. Differential screening of virus changing during the washing process. (A) Changes of virus types. (B) Changes of metagenomic NGS reads number. ( $C$ and D) Changes of the same virus in the washed fecal supernatant. $(E)$ Top ten viruses with the most significant fold change.

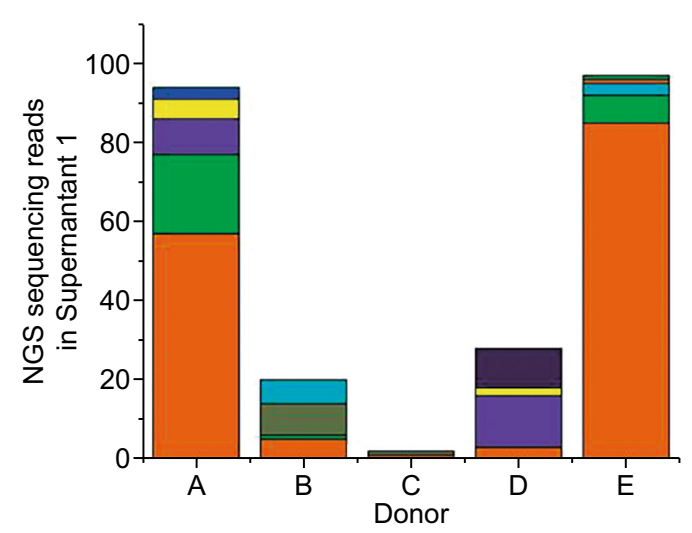

Pepper mild mottle virus

Tobacco mild green mosaic virus

- Garlic lalenl virus

Oryza saliva endornavirus

- Brassica yellows virus

- Tomalo mosaic virus

- Melon aphid borne yellows virus

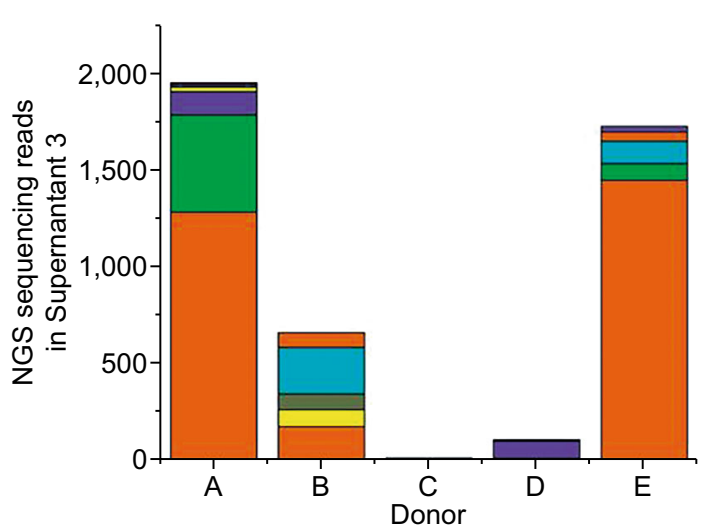

Walermelon mosaic virus

Cucumber green mottle mosaic virus

Apple stem pitting virus

Spinach lalenl virus

Elm mottle virus

Cilrus variegalion virus

Plodia inlerpunclella granulovirus

Figure 7. Top five viruses in each donor. 


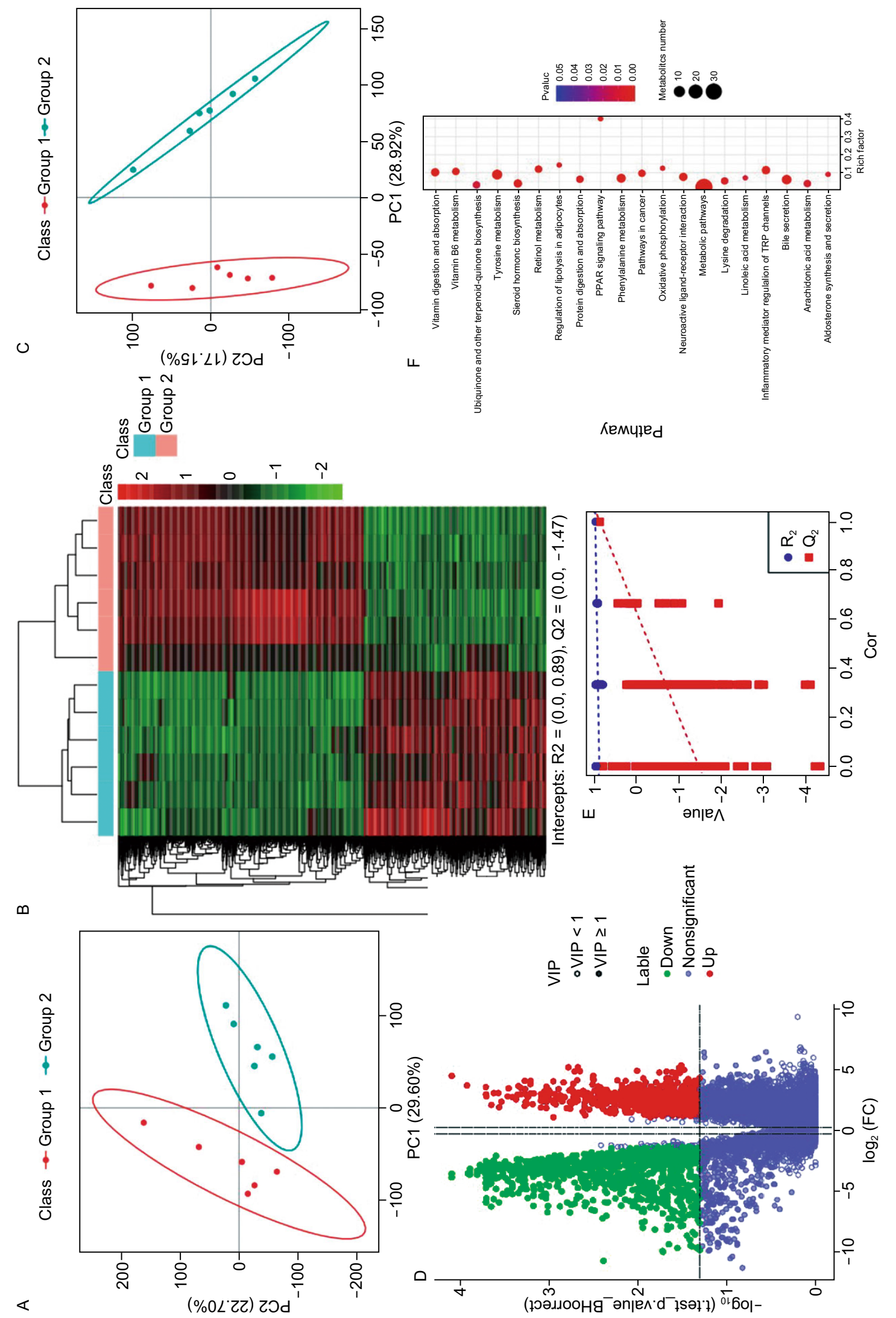

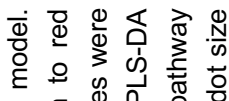
页

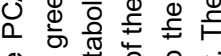
$\stackrel{\oplus}{=} \stackrel{0}{*}$

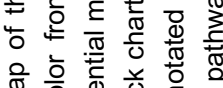

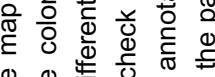
凹

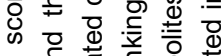
ष $\frac{\pi}{5}$ 需

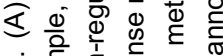

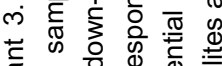

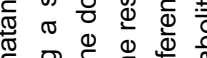

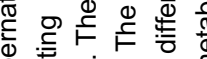

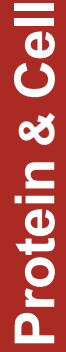


A

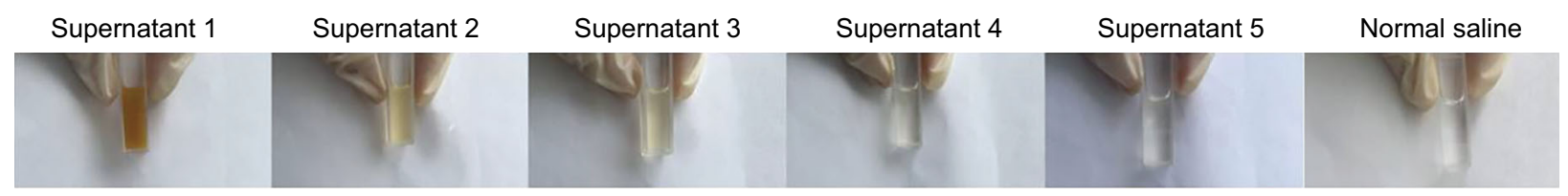

B

Exit surface


Figure 9. Differential testing by near-infrared absorption (NIRS) spectroscopy. (A) Incident surface of Supernatant 1, 2, 3, 4, 5 and normal saline. (B) Exit surface of Supernatant 1, 2, 3, 4, 5 and normal saline. (C) The light intensity of fecal microbiota supernatant by near-infrared absorption spectroscopy. (D) The absorbance of fecal microbiota supernatant by near-infrared absorption spectroscopy. (E) Euclidean distance between the spectrum of the supernatant of different washing times and the spectrum of the normal saline. (F) Correlation coefficient between the spectrum of the supernatant of different washing times and the spectrum of the normal saline.

Supernatant 1 exhibited the greatest absorbance compared to the other groups in each donor. And the absorbance showed a declining trend with the increase of washing times, which was closer to that of the normal saline. After repeated centrifugation plus suspension based on the automatic purification system, both light intensity and absorbance of Supernatant 5 were close to those of the normal saline. The analysis based on the euclidean distance and correlation coefficient demonstrated that the difference of light intensity between the supernatant and saline was getting smaller as the washing times increasing $(P<0.001)$.

\section{DISCUSSION}

The present study from population evidence showed that washed microbiota preparation significantly decreased FMT- related $A E s$ in $U C$ and $C D$ as compared with the manual preparation. Undigested food residues, fungi, parasite eggs and some small particles can be removed by the microfiltration system. The followed washing process washed out a certain bacterial fragments, metabolites, soluble molecules, and proteins. The above physical washing process contributed to the decreased rate of WMT-related AEs. Further animal experiments and in vitro differential screening supported the evidences for linking the clinical findings of the safety of FMT.

The washing microbiota method makes precise dose of fecal microbiota administration feasible. Up to now, most researches and consensuses recommend doctors to determine the therapeutic dose of microbiota based on the donor's fecal weight (Mattila et al., 2012; Satokari et al., 2015; Costello et al., 2016). This study first time reported that 
the fecal weight was not well correlated with the amount of enriched microbiota. This phenomenon can be observed among donors of different ages, and even different donations in one same donor. In terms of therapeutic dose, many studies recommend a single dose of $30-50 \mathrm{~g}$ fecal material to treat patients (Mattila et al., 2012; Satokari et al., 2015; Costello et al., 2016). The latest international consensus in 2019 suggested that the minimum quantity of donor stool is $12.5 \mathrm{~g}$ for upper gastrointestinal delivery, and $25 \mathrm{~g}$ for lower gastrointestinal delivery (Cammarota et al., 2019). The consensus also proposed that the ideal dose using fecal weight for clinical treatment remains uncertainty (Cammarota et al., 2019). In our center, we delivered a precise dose of microbiota based on the amount of enriched bacteria. This protocol might be one of the key factors resulting in satisfactory clinical efficacy in indications beyond CDI. Notably, a higher speed and a longer time were not used in current experimental conditions for centrifuging and washing bacteria for potentially saving living bacteria.

From the animal experiment, we found that the Supernatant 1 caused $70 \%$ of death in mice during 16-24 $\mathrm{h}$ after intraperitoneal injection. The remaining $30 \%$ of mice were very weak with chills. Meanwhile, no death was observed in other groups. The mice injected with the Supernatant 3 and 4 had the mildest and similar toxic reaction at both 6 and $24 \mathrm{~h}$ after intraperitoneal injection, respectively. The peripheral blood indexes of the Supernatant 3 were found to be close to the normal saline group, including WBC, RBC, PLT, percentage of NEUT and LYM. Further analysis indicated that no significant difference in blood cell counts between the third and fourth supernatant was found. Four times of centrifugation plus suspension may not be needed. These results from animal experiments verified the protocol in our FMT center using three times of centrifugation plus suspension after microfiltration. Then, the Supernatant 1 and Supernatant 3 were used for differential studies. Notably, the Supernatant 1 obtained after microfiltration plus centrifugation should be reasonably better than that from the manually prepared fecal suspension after crude filtration by using sterile dressings or kitchen strainer. This experimental mouse model was designed to amplify the host response to materials by intraperitoneal injection. This might be a promising animal model for evaluating the toxicity of materials which might be not easily detected by delivering them through intestinal tract.

In the hematoxylin-eosin (HE) staining assay for the spleen of mice, the proliferation of germinal center in secondary lymphoid follicles was only observed in the group of Supernatant 1, which corresponds with its serious sepsis (Pilgrim et al., 2007). This immune response may be related to the stimulation of bacterial fragments and their metabolites, as well as some small soluble molecules in the supernatant. These materials can act as antigens leading to sepsis. The obvious NEUT infiltration was also observed in the group of Supernatant 1 . The results further support that the toxic role of Supernatant 1 is stronger than Supernatant 3.

The number of WBC in the group of Supernatant 1 decreased significantly both at $6 \mathrm{~h}$ and $24 \mathrm{~h}$ after injection compared with the normal group of normal saline. These results indicated that the decline of WBC in mice might be a reflection of sepsis exacerbation in this study. This may be explained by the fact that these mice occur lipopolysaccharide (LPS)-induced endotoxemia and systemic inflammatory response syndrome (SIRS) after intraperitoneal injection with the fecal microbiota supernatant. In the fecal supernatant injected into mice there should be a certain bacteria fragments and their metabolites, small soluble molecules, as well as proteins washed out during the protocol for preparation of washed microbiota. As is known, Gram-negative bacteria account for a large percent of the bacteria in the gastrointestinal tract (Suffredini and Noveck, 2014). The fecal supernatant after repeated washing may play a toxic role in the host cells through the action of LPS from Gramnegative bacteria. LPS, a component of the outer wall of Gram-negative bacteria and also a kind of endotoxin, could activate a series of signal transduction pathways through the Toll-like receptors on the surface of the cell membrane to promote the occurrence of inflammation when it acted on the host cell (Jilma-Stohlawetz et al., 2017). Kurhaluk et al. established an endotoxemia model of mice (Kurhaluk et al., 2018) and observed an overall downward trend of WBC in the group of LPS. In addition, Yates et al. (2011) also found that in the LPS-induced ewes model, the number of WBC decreased significantly within $6 \mathrm{~h}$ after injection compared with the baseline. These reports are consistent with our findings in this study.

The group of Supernatant 1 showed a significantly decreased number of WBC and an increased percentage of NEUT at $24 \mathrm{~h}$ compared with that at $6 \mathrm{~h}$. This change may explain the increased mortality of $70 \%$ in the group of Supernatant 1 . The safety of the Supernatant 3 used in clinical actually was verified again in the mice model.

To investigate what was washed out after the last centrifugation, we performed metagenomic NGS analysis on the virus in the Supernatant 1 and Supernatant 3 from five healthy donors. As a result, we found a total of 34 types of viruses. Interestingly, all of these detected viruses (AboulAta et al., 2014; Symonds et al., 2018) from donors might be left-over from plant-based food. However, the previous researches in virome are limited. The further identification should be performed in the future to confirm whether these viruses were from human or not. The type and sequencing read of the virus were found to be different among the five donors, suggesting a possible role for the diet. Only one virus named pepper mild mottle virus was present in all five donors, and its sequencing reads increased consistently in the Supernatant 3 as compared with the Supernatant 1 . Pepper mild mottle virus as a plant pathogen might be a useful index virus for enteric viruses in monitoring the microbial quality of fresh produce and shellfish (Symonds 
et al., 2018). Similarly, Tobacco mild green mosaic virus was present in four donors, and its sequencing reads were also elevated in the Supernatant 3. Tobacco mild green mosaic virus coat protein could be used as an expression vector for the mimotope to be expressed into tobacco plants. Then this expressed recombinant protein, as an edible vaccine, had both a therapeutic and a diagnostic role (Aboul-Ata et al., 2014). Consistently, the total viral types and sequencing number of the five donors increased in the Supernatant 3 as compared with the Supernatant 1 . The results indicate the viruses could be eluted from the microbiota into the supernatant in normal saline as a vector solution, especially for Watermelon mosaic virus, Cherry green ring mottle virus and Paprika mild mottle virus.

The differential metabolites with pro-inflammatory effects were found to be mainly washed out in the Supernatant 1 , including LtB4, CORT, PGG2, and 5-HIAA. Kwon et al. (2019) found that LtB4 and its receptors (BLT1 and BLT2) acting as inflammatory lipid mediators aggravated LPS-induced endotoxic shock in mice. CORT was (Lin et al., 2019) confirmed to be involved in the neuroinflammatory response in the LPS-induced sepsis model. As is known to all, prostaglandins (PGs) as a metabolite of arachidonic acid play an important role in fever, inflammation and blood pressure regulation. PGG2 was an intermediate metabolite which can be further transferred into different PGs (such as PGI2, PGE2, PGF2 $\alpha, P G D 2$ ) through prostaglandin synthase (Alhouayek and Muccioli, 2014). The increased PGG2 can increase pro-inflammatory macrophage activation induced by LPS in vitro (Alhouayek et al., 2013). The concentration of 5-HIAA was reported to be associated with highly sensitive C-reactive protein, which was a marker of chronic low-grade inflammation in metabolic syndrome (Afarideh et al., 2015). Hence, the presence of these pro-inflammatory substances may explain the aggravation of inflammation and even death in mice injected with the Supernatant 1. Meanwhile, several significantly enriched metabolic pathways played an important role in the inflammatory reaction. The pathway of inflammatory mediator regulation of TRP channels plays different roles in inflammatory response due to it consists of more than 30 members which can be divided into 7 subfamilies (TRPC, TRPV, TRPM, TRPA, TRPP, TRPML, TRPN). TRPMs can inhibit the secretion of anti-inflammatory cytokine (Nilius and Owsianik, 2011) and participate in the inflammatory process through the antagonism against neutrophils (Wang et al., 2014). Boltana et al. (2018) found that TRPVs participated in the process of temperature up-regulation concomitantly with other pro-inflammatory cytokines such as PGE2, TNF- $\alpha$, IL-6, and IL-1 $\beta$.

In Supernatant 3 , a total of 13 anti-inflammatory metabolites were noted. Many of them can inhibit the activation of the LPS-induced NF-KB signaling pathway to reduce the inflammatory response, including tretinoin (Austenaa et al., 2009), stearidonic acid (Sung et al., 2017), caffeic acid (Kim et al., 2014), tyrosol (Lu et al., 2013), aspirin (Liu et al., 2017), 4-hydroxy-2-nonenal (Kim et al.,
2009). Resveratrol (Bigagli et al., 2017), chloral hydrate (Cai et al., 2016) and paracetamol (Vuong et al., 2019) have antiinflammatory and anti-oxidant effects in LPS-stimulated RAW 264.7 macrophage cells in vitro. Calcitriol, the active form of vitamin $D$, has been confirmed that it can reduce the infiltration of inflammatory cells and attenuate the elevation of TNF- $\alpha$ during LPS-induced acute lung injury in mice (Tan et al., 2016). Similarly, in the lung injury model of mice (Metsola et al., 2014), adenosine regulates endothelial permeability and plays a role of anti-inflammation via its receptors. These anti-inflammatory metabolites increased in the Supernatant 3 may explain why the Supernatant 3 induced inflammation was less than that of the Supernatant 1. This should contribute to decreasing the rate of clinical AEs of microbiota transplant. Additionally, the process of washing microbiota does loss some beneficial molecules, such as anti-inflammation or anti-oxidant elements, the efficacy was not affected in UC (Ding et al., 2019) and CD (Wang et al., 2018).

The experiment using NIRS is a way to seek the standard of using the optical spectrum technique to control the quality of the washing process. The qualified final washed samples are based on the standard process. However, further study is necessary to determine whether this method can be widely used in practice.

There are some limitations in this study. More inflammatory markers tested in the mouse experiment should be more solid. In the metagenomic NGS for virus screening, not all genomes are available. The differential screening for enriched microbiota would be supportive of clarifying the value of washed microbiota preparation protocol.

In conclusion, this study first time provides the evidence from clinical findings, animal experiments and in vitro tests to support that the protocol of WMT is better than the manual preparation of FMT in improving safety, enriching the precise amount of microbiota and quality controllable in practice. Our findings might encourage more researches to use this novel preparation to enrich microbiota from feces in laboratory study and move the crude FMT into the stage of WMT in practice.

\section{MATERIALS AND METHODS}

\section{Data analysis on FMT-related AEs in IBD}

The potential factors related to AEs were analyzed in patients with IBD who underwent FMT (Fig. 1). All data were from CMTS (www. fmtbank.org), which was supported by China National Clinical Research Center for Digestive Diseases (Xi'an) for a long-term evaluation on the decision, treatment, efficacy and safety of microbiota transplant. The data related to FMT was collected from Oct 25, 2012 to Oct 10,2019 by searching the database, which follows the ethical protocol of CMTS. The clinical data of this clinical study was based on our pooled registered trials (NCT01790061, NCT01793831 and NCT02998112) in clinicaltrails.gov. AEs were confirmed by the international guideline Common Terminology 
Criteria for Adverse Events (CTCAE) version 5.0 as our previous reports (Wang et al., 2018; Ding et al., 2019). All trials were approved by the institutional review board of the Second Affiliated Hospital of Nanjing Medical University and the animal ethics committee of Nanjing Medical University. Written informed consent was obtained from all subjects.

\section{Fecal microbiota preparation}

All recorded data on the weight of donated feces, the time for donation and the age of donors were collected for analysis (Fig. 1). Donors were recruited according to the long-term criteria for donor selection at our center (Ding et al., 2019; Zhang et al., 2019). Briefly, we used the eight criteria for screening a donor, including age, physiology, pathology, psychology, honesty, time, environment and recipient state (Ding et al., 2019; Zhang et al., 2019).

The method for preparation of microbiota is based on the automatic microbiota purification system followed with centrifugation plus suspension for three times in a specially designed exclusive laboratory at good manufacture practice (GMP) level (Cui et al., 2016; Zhang et al., 2018). The fecal particles, parasites eggs and fungus in the fecal suspension are removed by sequential microfiltration based on the automatic machine. The "one-hour FMT protocol" limited the time from defecation of a donor, laboratory preparation for enriching microbiota to the time of microbiota delivering, or microbiota storing within one hour (He et al., 2017; Zhang et al., 2018; Ding et al., 2019). The donated feces were collected using a disposable bottle matching the GenFMTer. The ratio of $500 \mathrm{~mL}$ saline per $100 \mathrm{~g}$ feces was used for homogeneously making fecal suspension, and then the scheduled microfiltration within the machine was automatically processed. The fecal microbiota suspension after microfiltration was automatically dispersed into serial 50-mL tubes for the following centrifugation for 3 $\mathrm{min}$ at a speed of $700 \times g(2000 \mathrm{rpm}$, TDZ5-WS, XIANGZHI, Changsha, China). The supernatant obtained after centrifugation of the fecal microbiota suspension was suctioned. The same subsequent centrifugation was performed after $0.9 \%$ sodium chloride was added to the microbiota precipitation. Briefly, the fecal supernatant after the first to fifth centrifugation plus suspension was collected for animal experiments or metabolism and virus tests. The fecal supernatant obtained after filtration from GenFMTer following the first centrifugation was defined as Supernatant 1. The fecal supernatant after the repeated centrifugation plus suspension for two, three, four and five times was defined as Supernatant 2, Supernatant 3 , Supernatant 4 , and Supernatant 5, respectively. The study flow was shown in Fig. 1.

\section{Mouse model by intraperitoneal injection}

All experimental protocols were reviewed and approved by the animal ethics committee of Nanjing Medical University (No. IACUC1910004). The male C57BL/6 mice weighing 17-20 g from Animal Center of Nanjing Medical University were used. The mice were injected intraperitoneally with the fecal supernatant at the dose of 0.3 $\mathrm{mL}$ per mouse. The control group was injected with the same dose of $0.9 \%$ sodium chloride. The observation points are set at $6 \mathrm{~h}$ after injection, time of death, and $24 \mathrm{~h}$ after injection if still alive. We took peripheral blood from the orbit for routine analysis of blood and pathological sections of the spleen to observe the infiltration of inflammatory cells. Blood samples from retro-orbital plexus were collected in an anticoagulant tube containing EDTAK2 (KANG JIAN, China) and analyzed using Automatic Hematology Analyzer (Hisenmekang-XN550, Japan). No significant differences in sepsis of mice were observed between Supernatant 3 and Supernatant 4 as well as Supernatant 5 . Then, the Supernatant 1 , Supernatant 2 , Supernatant 3 and Supernatant 4 were selected for the next experiments. The spleen tissues were taken and soaked in $4 \%$ paraformaldehyde for a paraffin slice and HE staining. Neutrophil infiltration and proliferation of follicles were evaluated and graded. The number of neutrophils in the visual field differed from scattered to plentiful was classified as "+-+++". "+" means scattered neutrophils, while "++" or "+++" means moderate and plentiful number of neutrophils, respectively. Follicles included primary follicles and the secondary follicles. The classification of the follicles proliferation was none or existing. These experiments further confirmed that the Supernatant 1 and Supernatant 3 were collected for further differential screening on viruses and metabolites.

\section{Virus screening by metagenomic NGS}

Total $1.5 \mathrm{~mL}$ of Supernatant 1 and 3 were collected for nucleotides purification with TIANamp Micro DNA Kit (DP316, TIANGEN BIOTECH) for DNA extraction and Vision Medicals Cat (VM001, Guangzhou, China) for RNA extraction according to the manufacturer's recommendation (Miller et al., 2019). DNA was sheared under power 50 for $155 \mathrm{~s}$ by using focused-ultrasonicators (Covaris). In total, 100 ng sheared DNA was subjected to library construction with VAHTS ${ }^{\text {TM }}$ Universal DNA Library Prep kit (ND 607, VAZYME BIOTECH). After purification and sorting, Agilent 2100 bioanalyzer was used to profile the DNA length of the library, which was purified and sorted. Library with $300 \pm 50$ bp peak passed and was subjected to library pooling. Before being subjected to library preparation, concentrations of extracted DNA/RNA were measured by a Qubit Fluorometer. Human rRNA molecules from the RNA samples were depleted by an RNase $\mathrm{H}$-based method (Vision Medicals Cat\# VM003, Guangzhou, China). DNA library was prepared by a transposase-based methodology.

Illumina NextSeq550 sequencers were used to sequence by using a $75 \mathrm{bp}$, single-end and single index sequencing kit (Illumina Cat\#CN500, San Diego). Each sample can obtain approximately 20 million reads. High-quality sequencing data were generated after excluding low-quality and short (length $<35 \mathrm{bp}$ ) reads, and human host sequences were subtracted, which were mapped to the human reference genome (hg39) and plasmids using Burrows-Wheeler alignment (Li and Durbin, 2009). After the removal of low complexity reads, the remaining data were mapped to the IDseq microbial genome databases consisting of viruses, bacteria, fungi, and parasites. The taxonomic references were downloaded from the National Center Biotechnology Information (ftp://ftp.ncbi.nlm.nih.gov/ genomes/ftp://ftp.ncbi.nlm.nih.gov/genomes/). Upon identification of critical pathogen, the identified species-specific sequences were further confirmed by Blastn to validate its accuracy (http://www.blast. ncbi.nlm.nih.gov/Blast.cgi). The experiment was performed by Vision Medicals company (Guangzhou, China). 


\section{Metabolism analysis by LC-MS/MS}

The supernatant samples were stored at $-80^{\circ} \mathrm{C}$ before being sent to the company (BGI, Wuhan) for analysis. Methanol was used for extraction for MS analysis. After repeated grinding $(50 \mathrm{~Hz}, 5 \mathrm{~min})$ and centrifuging $(25,000 \mathrm{rpm}, 15 \mathrm{~min})$, metabolites were extracted for liquid chromatography-mass spectrometry analysis. Waters 2D UPLC (Waters, USA) tandem Q Exactive high-resolution mass spectrometer (Thermo Fisher Scientific, USA) was used for the separation and detection of metabolites. The chromatographic column used was BEHC18 column $(1.7 \mu \mathrm{m} 2.1 \times 100 \mathrm{~mm}$, Waters, USA). The running mode of LC-MS was called the binary gradient solvent mode, consisting of $0.1 \%(v / v)$ formic acid in water (solvent $A$ ) and $0.1 \%$ formic acid in $100 \%$ methanol (solvent $B$ ) in the positive ion mode and $10 \mathrm{mmol} / \mathrm{L}$ formic acid ammonia in water (solvent A) and $10 \mathrm{mmol} / \mathrm{L}$ formic acid ammonia in 95\% methanol (solvent $B$ ) in negative ion mode. We used HMDB, KEGG and LipidMaps database to analyze the data.

\section{Spectrum analysis}

In order to quickly confirm the quality of the washing process for enriching microbiota from feces, NIRS was used to perform the differential test for each supernatant after centrifugation. The QE Pro high-performance spectrometer (Ocean Optics Inc. USA) and HL2000-LL light source (Ocean Optics Inc. USA) were used to setup the measurement system. The effective detection wavelength range was set as 300-950 nm. Solution sample were added into a cuvette for tests. Each sample was measured for 10 times, repeatedly, to verify the stability of the experimental system. The spectral curves of each sample would be obtained for analysis. The software-supported system was set by the Biomedical Engineering Department, TianGong University, China.

\section{Statistical analysis}

Data were analyzed and performed using SPSS (Chicago, IL, USA) and GraphPad Prism (La Jolla, CA, USA). When the normality of the distribution of variables was acceptable, the unpaired Student's $t$ test and one-way ANOVA were used to analyze differences between groups. Otherwise, the nonparametric test was used. Categorical variables were analyzed by chi-square test. Pearson correlation was used for correlation analysis. Differences were considered significant when $P<0.05$. The $q$ value is obtained after the false discovery rate (FDR) correction of the $P$ value.

\section{ACKNOWLEDGMENTS}

The authors would like to appreciate the kindly help from Jie Zhang for providing data from China Microbiota Transplantation System (http://www.fmtbank.org).

\section{ABBREVIATIONS}

$\mathrm{AE}$, adverse event; $\mathrm{CDI}$, Clostridioides difficile infection; $\mathrm{CD}$, Crohn's disease; CORT, corticosterone; CMTS, China Microbiota Transplantation System; FDR, false discovery rate; FMT, fecal microbiota transplantation; GMP, good manufacture practice; KEGG, Kyoto encyclopedia of genes and genomes; LYM, lymphocyte; LtB4, leukotriene B4; LPS, lipopolysaccharide; NGS, next-generation sequencing; NIRS, near-infrared absorption spectroscopy; NEUT, neutrophil; NLR, NEUT to LYM ratio; PLT, platelet; PLR, PLT to LYM ratio; PCA, principle component analysis; PGs, prostaglandins; PLSDA, partial least squares method-discriminant analysis; PGG2, prostaglandin G2; RBC, red blood cell; RCT, randomized controlled trial; SIRS, systemic inflammatory response syndrome; TRP, transient receptor potential; UC, ulcerative colitis; WBC, white blood cell; WMT, washed microbiota transplantation.

\section{AUTHOR CONTRIBUTIONS}

T.Z., G.L. and Z.Z. designed and performed experiments, analyzed data, and wrote the manuscript. Y.L., P.L., Y.C. and C.M. completed the animal experiments. P.L. performed bacteria counting. Z.Z., H.Y. and H.W. performed the spectrum experiment. B.C., G.J., Q.S. and L.C. joined the experiments. F.Z. conceived the idea of WMT, designed the experiments, and wrote the manuscript.

\section{FUNDING}

This work was supported by publicly donated Intestine Initiative Foundation; Primary Research \& Development Plan of Jiangsu Province (BE2018751); Jiangsu Provincial Medical Innovation Team (Zhang F), National Natural Science Foundation of China (81600417, 81670495 and 81873548), and China National Center for Clinical Research of Digestive Diseases (201502026).

\section{COMPLIANCE WITH ETHICS GUIDELINES}

Faming Zhang invented the concept of GenFMTer, TET and the related devices. Ting Zhang, Gaochen Lu, Zhe Zhao, Yafei Liu, Quan Shen, Pan Li, Yaoyao Chen, Haoran Yin, Huiquan Wang, Cicilia Marcella, Bota Cui, Lei Cheng, and Guozhong Ji declare that they have no competing interests. All procedures followed were in accordance with the ethical standards of the institutional review board of the Second Affiliated Hospital of Nanjing Medical University and with the Helsinki Declaration of 1975, as revised in 2000. Informed consent was obtained from all patients for being included in the study. All institutional and national guidelines for the care and use of laboratory animals were followed.

\section{OPEN ACCESS}

This article is licensed under a Creative Commons Attribution 4.0 International License, which permits use, sharing, adaptation, distribution and reproduction in any medium or format, as long as you give appropriate credit to the original author(s) and the source, provide a link to the Creative Commons licence, and indicate if changes were made. The images or other third party material in this article are included in the article's Creative Commons licence, unless indicated otherwise in a credit line to the material. If material is not included in the article's Creative Commons licence and your intended use is not permitted by statutory regulation or exceeds the permitted use, you will need to obtain permission directly from the copyright holder. To view a copy of this licence, visit http:// creativecommons.org/licenses/by/4.0/. 


\section{REFERENCES}

Aboul-Ata AA, Vitti A, Nuzzaci M, El-Attar AK, Piazzolla G, Tortorella C, Harandi AM, Olson O, Wright SA, Piazzolla P (2014) Plantbased vaccines: novel and low-cost possible route for Mediterranean innovative vaccination strategies. Adv Virus Res 89:1-37

Afarideh M, Behdadnia A, Noshad S, Mirmiranpour H, Mousavizadeh M, Khajeh E, Rad MV, Mazaheri T, Nakhjavani M, Esteghamati A (2015) Association of peripheral 5-hydroxyindole-3-acetic acid, a serotonin derivative, with metabolic syndrome and low-grade inflammation. Endocr Pract 21:711-718

Alhouayek M, Muccioli GG (2014) COX-2-derived endocannabinoid metabolites as novel inflammatory mediators. Trends Pharmacol Sci 35:284-292

Alhouayek M, Masquelier J, Cani PD, Lambert DM, Muccioli GG (2013) Implication of the anti-inflammatory bioactive lipid prostaglandin D2-glycerol ester in the control of macrophage activation and inflammation by ABHD6. Proc Natl Acad Sci U S A 110:17558-17563

Allegretti JR, Mullish BH, Kelly C, Fischer M (2019) The evolution of the use of faecal microbiota transplantation and emerging therapeutic indications. Lancet 394:420-431

Austenaa LM, Carlsen H, Hollung K, Blomhoff HK, Blomhoff R (2009) Retinoic acid dampens LPS-induced NF-kappaB activity: results from human monoblasts and in vivo imaging of NFkappaB reporter mice. J Nutr Biochem 20:726-734

Bajaj JS, Kassam Z, Fagan A, Gavis EA, Liu E, Cox IJ, Kheradman R, Heuman D, Wang J, Gurry T et al (2017) Fecal microbiota transplant from a rational stool donor improves hepatic encephalopathy: a randomized clinical trial. Hepatology 66:1727-1738

Bigagli E, Cinci L, Paccosi S, Parenti A, D’Ambrosio M, Luceri C (2017) Nutritionally relevant concentrations of resveratrol and hydroxytyrosol mitigate oxidative burst of human granulocytes and monocytes and the production of pro-inflammatory mediators in LPS-stimulated RAW 264.7 macrophages. Int Immunopharmacol 43:147-155

Boltana S, Sanhueza N, Donoso A, Aguilar A, Crespo D, Vergara D, Arriagada G, Morales-Lange B, Mercado L, Rey $S$ et al (2018) The expression of TRPV channels, prostaglandin E2 and proinflammatory cytokines during behavioural fever in fish. Brain Behav Immun 71:169-181

Brandt LJ (2012) Editorial commentary: fecal microbiota transplantation: patient and physician attitudes. Clin Infect Dis 55:16591660

Cai J, Peng Y, Chen T, Liao H, Zhang L, Chen Q, He Y, Wu P, Xie T, Pan $Q$ (2016) Chloral hydrate treatment induced apoptosis of macrophages via Fas signaling pathway. Med Sci Monit 22:4836-4843

Cammarota G, laniro G, Tilg $H$, Rajilic-Stojanovic M, Kump P, Satokari R, Sokol H, Arkkila P, Pintus C, Hart A et al (2017) European consensus conference on faecal microbiota transplantation in clinical practice. Gut 66:569-580

Cammarota G, laniro G, Kelly CR, Mullish BH, Allegretti JR, Kassam Z, Putignani L, Fischer M, Keller JJ, Costello SP et al (2019) International consensus conference on stool banking for faecal microbiota transplantation in clinical practice. Gut 68(12):21112121

Costello SP, Tucker EC, La Brooy J, Schoeman MN, Andrews JM (2016) Establishing a fecal microbiota transplant service for the treatment of Clostridium difficile infection. Clin Infect Dis 62:908914

Costello SP, Hughes PA, Waters O, Bryant RV, Vincent AD, Blatchford P, Katsikeros R, Makanyanga J, Campaniello MA, Mavrangelos $C$ et al (2019) Effect of fecal microbiota transplantation on 8-week remission in patients with ulcerative colitis: a randomized clinical trial. JAMA 321:156-164

Cui B, Li P, Xu L, Zhao Y, Wang H, Peng Z, Xu H, Xiang J, He Z, Zhang $T$ et al (2015) Step-up fecal microbiota transplantation strategy: a pilot study for steroid-dependent ulcerative colitis. J Transl Med 13:298

Cui B, Li P, Xu L, Peng Z, Xiang J, He Z, Zhang T, Ji G, Nie Y, Wu K et al (2016) Step-up fecal microbiota transplantation (FMT) strategy. Gut Microbes 7:323-328

DeFilipp Z, Bloom PP, Torres Soto M, Mansour MK, Sater MRA, Huntley MH, Turbett S, Chung RT, Chen YB, Hohmann EL (2019) Drug-resistant $E$. coli bacteremia transmitted by fecal microbiota transplant. N Engl J Med 381:2043-2050

Ding X, Li Q, Li P, Zhang T, Cui B, Ji G, Lu X, Zhang F (2019) Longterm safety and efficacy of fecal microbiota transplant in active ulcerative colitis. Drug Saf 42:869-880

He Z, Li P, Zhu J, Cui B, Xu L, Xiang J, Zhang T, Long C, Huang G, Ji $G$ et al (2017) Multiple fresh fecal microbiota transplants induces and maintains clinical remission in Crohn's disease complicated with inflammatory mass. Sci Rep 7:4753

Huang HL, Chen HT, Luo QL, Xu HM, He J, Li YQ, Zhou YL, Yao F, Nie YQ, Zhou YJ (2019) Relief of irritable bowel syndrome by fecal microbiota transplantation is associated with changes in diversity and composition of the gut microbiota. J Dig Dis 20:401408

Jilma-Stohlawetz P, Kliegel T, Kantner-Schlifke I, Strasser-Marsik C, Mayr FB, Jilma B (2017) Upregulation of cytokine mRNA in circulating leukocytes during human endotoxemia. Eur Cytokine Netw 28:19-26

Kang DW, Adams JB, Gregory AC, Borody T, Chittick L, Fasano A, Khoruts A, Geis E, Maldonado J, McDonough-Means $S$ et al (2017) Microbiota transfer therapy alters gut ecosystem and improves gastrointestinal and autism symptoms: an open-label study. Microbiome 5:10

Kim YS, Park ZY, Kim SY, Jeong E, Lee JY (2009) Alteration of Tolllike receptor 4 activation by 4-hydroxy-2-nonenal mediated by the suppression of receptor homodimerization. Chem Biol Interact 182:59-66

Kim SR, Jung YR, Kim DH, An HJ, Kim MK, Kim ND, Chung HY (2014) Caffeic acid regulates LPS-induced NF-kappaB activation through NIK/IKK and c-Src/ERK signaling pathways in endothelial cells. Arch Pharm Res 37:539-547

Konig J, Siebenhaar A, Hogenauer C, Arkkila P, Nieuwdorp M, Noren T, Ponsioen CY, Rosien U, Rossen NG, Satokari R et al (2017) Consensus report: faecal microbiota transfer-clinical applications and procedures. Aliment Pharmacol Ther 45:222239 
Kurhaluk N, Zaitseva OV, Sliuta A, Kyriienko S, Winklewski PJ (2018) Melatonin diminishes oxidative stress in plasma, retains erythrocyte resistance and restores white blood cell count after low dose lipopolysaccharide exposure in mice. Gen Physiol Biophys 37:571-580

Kwon SY, Ro M, Kim JH (2019) Mediatory roles of leukotriene B4 receptors in LPS-induced endotoxic shock. Sci Rep 9:5936

Li H, Durbin R (2009) Fast and accurate short read alignment with Burrows-Wheeler transform. Bioinformatics 25:1754-1760

Lin L, Chen X, Zhou Q, Huang P, Jiang S, Wang H, Deng Y (2019) Synaptic structure and alterations in the hippocampus in neonatal rats exposed to lipopolysaccharide. Neurosci Lett 709:134364

Liu Y, Fang S, Li X, Feng J, Du J, Guo L, Su Y, Zhou J, Ding G, Bai Y et al (2017) Aspirin inhibits LPS-induced macrophage activation via the NF-kappaB pathway. Sci Rep 7:11549

Lu J, Huang G, Wang Z, Zhuang S, Xu L, Song B, Xiong Y, Guan S (2013) Tyrosol exhibits negative regulatory effects on LPS response and endotoxemia. Food Chem Toxicol 62:172-178

Ma Y, Yang J, Cui B, Xu H, Xiao C, Zhang F (2017) How Chinese clinicians face ethical and social challenges in fecal microbiota transplantation: a questionnaire study. BMC Med Ethics 18:39

Mattila E, Uusitalo-Seppala R, Wuorela M, Lehtola L, Nurmi H, Ristikankare M, Moilanen V, Salminen K, Seppala M, Mattila PS et al (2012) Fecal transplantation, through colonoscopy, is effective therapy for recurrent Clostridium difficile infection. Gastroenterology 142:490-496

McDonald LC, Gerding DN, Johnson S, Bakken JS, Carroll KC, Coffin SE, Dubberke ER, Garey KW, Gould CV, Kelly C et al (2018) Clinical practice guidelines for Clostridium difficile infection in adults and children: 2017 update by the Infectious Diseases Society of America (IDSA) and Society for Healthcare Epidemiology of America (SHEA). Clin Infect Dis 66:987-994

McSweeney B, Allegretti JR, Fischer $M, X u H$, Goodman KJ, Monaghan T, McLeod C, Mullish BH, Petrof EO, Phelps EL et al (2019) In search of stool donors: a multicenter study of prior knowledge, perceptions, motivators, and deterrents among potential donors for fecal microbiota transplantation. Gut Microbes. https://doi.org/10.1080/19490976.2019.1611153

Metsola J, Maksimow M, Ojaniemi M, Metsola H, Marttila-Ichihara F, Vuolteenaho R, Yegutkin GG, Salmi M, Hallman M, Jalkanen S (2014) Postnatal development and LPS responsiveness of pulmonary adenosine receptor expression and of adenosinemetabolizing enzymes in mice. Pediatr Res 76:515-521

Miller S, Naccache SN, Samayoa E, Messacar K, Arevalo S, Federman S, Stryke D, Pham E, Fung B, Bolosky WJ et al (2019) Laboratory validation of a clinical metagenomic sequencing assay for pathogen detection in cerebrospinal fluid. Genome Res 29:831-842

Moayyedi P, Surette MG, Kim PT, Libertucci J, Wolfe M, Onischi C, Armstrong D, Marshall JK, Kassam Z, Reinisch W et al (2015) Fecal microbiota transplantation induces remission in patients with active ulcerative colitis in a randomized controlled trial. Gastroenterology 149(102-109):e106

Ng SC, Kamm MA, Yeoh YK, Chan PKS, Zuo T, Tang W, Sood A, Andoh A, Ohmiya N, Zhou $Y$ et al (2019) Scientific frontiers in faecal microbiota transplantation: joint document of Asia-Pacific
Association of Gastroenterology (APAGE) and Asia-Pacific Society for Digestive Endoscopy (APSDE). Gut 69(1):83-91

Nilius B, Owsianik G (2011) The transient receptor potential family of ion channels. Genome Biol 12:218

Paramsothy S, Kamm MA, Kaakoush NO, Walsh AJ, van den Bogaerde J, Samuel D, Leong RWL, Connor S, Ng W, Paramsothy $R$ et al (2017) Multidonor intensive faecal microbiota transplantation for active ulcerative colitis: a randomised placebocontrolled trial. Lancet 389:1218-1228

Park L, Mone A, Price JC, Tzimas D, Hirsh J, Poles MA, Malter L, Chen LA (2017) Perceptions of fecal microbiota transplantation for Clostridium difficile infection: factors that predict acceptance. Ann Gastroenterol 30:83-88

Pilgrim MJ, Kasman L, Grewal J, Bruorton ME, Werner P, London L, London SD (2007) A focused salivary gland infection with attenuated MCMV: an animal model with prevention of pathology associated with systemic MCMV infection. Exp Mol Pathol 82:269-279

Qi X, Li X, Zhao Y, Wu X, Chen F, Ma X, Zhang F, Wu D (2018) Treating steroid refractory intestinal acute graft-vs.-host disease with fecal microbiota transplantation: a pilot study. Front Immunol 9:2195

Satokari R, Mattila E, Kainulainen V, Arkkila PE (2015) Simple faecal preparation and efficacy of frozen inoculum in faecal microbiota transplantation for recurrent Clostridium difficile infection-an observational cohort study. Aliment Pharmacol Ther 41:46-53

Sood A, Mahajan R, Juyal G, Midha V, Grewal CS, Mehta V, Singh A, Joshi MC, Narang V, Kaur K et al (2019) Efficacy of fecal microbiota therapy in steroid dependent ulcerative colitis: a real world intention-to-treat analysis. Intest Res 17:78-86

Suffredini AF, Noveck RJ (2014) Human endotoxin administration as an experimental model in drug development. Clin Pharmacol Ther 96:418-422

Sung J, Jeon H, Kim IH, Jeong HS, Lee J (2017) Anti-inflammatory effects of stearidonic acid mediated by suppression of NFkappaB and MAP-kinase pathways in macrophages. Lipids 52:781-787

Symonds EM, Nguyen KH, Harwood VJ, Breitbart M (2018) Pepper mild mottle virus: a plant pathogen with a greater purpose in (waste)water treatment development and public health management. Water Res 144:1-12

Tan ZX, Chen YH, Xu S, Qin HY, Wang H, Zhang C, Xu DX, Zhao H (2016) Calcitriol inhibits tumor necrosis factor alpha and macrophage inflammatory protein-2 during lipopolysaccharideinduced acute lung injury in mice. Steroids 112:81-87

Vuong LD, Nguyen QN, Truong VL (2019) Anti-inflammatory and anti-oxidant effects of combination between sulforaphane and acetaminophen in LPS-stimulated RAW 264.7 macrophage cells. Immunopharmacol Immunotoxicol 41:413-419

Wang CH, Rong MY, Wang L, Ren Z, Chen LN, Jia JF, Li XY, Wu ZB, Chen ZN, Zhu P (2014) CD147 up-regulates calcium-induced chemotaxis, adhesion ability and invasiveness of human neutrophils via a TRPM-7-mediated mechanism. Rheumatology (Oxford) 53:2288-2296

Wang H, Cui B, Li Q, Ding X, Li P, Zhang T, Yang X, Ji G, Zhang F (2018) The safety of fecal microbiota transplantation for Crohn's disease: findings from a long-term study. Adv Ther 35:1935-1944 
Wang JW, Wang YK, Zhang F, Su YC, Wang JY, Wu DC, Hsu WH (2019) Initial experience of fecal microbiota transplantation in gastrointestinal disease: a case series. Kaohsiung J Med Sci 35:566-571

Wu X, Dai M, Buch H, Bai J, Long W, Long C, Tang X, Tu H, Zhang $\mathrm{R}$, Zhu $\mathrm{C}$ et al (2019) The recognition and attitudes of postgraduate medical students toward fecal microbiota transplantation: a questionnaire study. Therap Adv Gastroenterol 12:1756284819869144

Yates DT, Loest CA, Ross TT, Hallford DM, Carter BH, Limesand SW (2011) Effects of bacterial lipopolysaccharide injection on white blood cell counts, hematological variables, and serum glucose, insulin, and cortisol concentrations in ewes fed low- or highprotein diets. J Anim Sci 89:4286-4293

Zhang F, Luo W, Shi Y, Fan Z, Ji G (2012) Should we standardize the 1,700-year-old fecal microbiota transplantation? Am J Gastroenterol 107:1755-1756
Zhang F, Cui B, He X, Nie Y, Wu K, Fan D, FMT-standardization Study Group (2018) Microbiota transplantation: concept, methodology and strategy for its modernization. Protein Cell 9:462-473

Zhang F, Zhang T, Zhu H, Borody TJ (2019) Evolution of fecal microbiota transplantation in methodology and ethical issues. Curr Opin Pharmacol 49:11-16

Zipursky JS, Sidorsky TI, Freedman CA, Sidorsky MN, Kirkland KB (2012) Patient attitudes toward the use of fecal microbiota transplantation in the treatment of recurrent Clostridium difficile infection. Clin Infect Dis 55:1652-1658

Zipursky JS, Sidorsky TI, Freedman CA, Sidorsky MN, Kirkland KB (2014) Physician attitudes toward the use of fecal microbiota transplantation for the treatment of recurrent Clostridium difficile infection. Can J Gastroenterol Hepatol 28:319-324 